\title{
Correction to: Nexus between agro-ecological efficiency and carbon emission transfer: evidence from China
}

\author{
Usman Akbar ${ }^{1} \cdot$ Quan-Lin $\mathrm{Li}^{2} \cdot$ Muhammad Abdullah Akmal $^{3} \cdot$ Mohammed Shakib $^{1} \cdot$ Wasim Iqbal $^{4}$ (D \\ Published online: 2 July 2021 \\ (C) Springer-Verlag GmbH Germany, part of Springer Nature 2021
}

\section{Correction to: Environmental Science and Pollution Research (2021) 28:18995-19007} https://doi.org/10.1007/s11356-020-09614-2

The correct affiliations of the Authors are presented in this paper.

Publisher's note Springer Nature remains neutral with regard to jurisdictional claims in published maps and institutional affiliations.

The online version of the original article can be found at https://doi.org/ 10.1007/s11356-020-09614-2

Usman Akbar

usman.akbar@stumail.ysu.edu.cn; usman.akbar@outlook.com

Quan-Lin Li

liquanlin@tsinghua.edu.cn

Muhammad Abdullah Akmal

agfa.94@gmail.com; abdullah.akmal@giki.edu.pk

Mohammed Shakib

shakibbd@gmail.com

Wasim Iqbal

wasimiqbal01@yahoo.com
School of Economics and Management, Yanshan University, 438, Hebei Avenue, Haigang District, Qinhuangdao City 066004, Hebei, People's Republic of China

2 School Of Economics And Management, Beijing University of Technology, Beijing, China

3 Faculty Of Computer Science and Engineering, Ghulam Ishaq Khan Institution Of Engineering Sciences and Technology, Topi 23640, Pakistan

4 College of Economics and Management, Shenzhen University, Shenzhen, Guangdong Province, China 